\title{
Cardiac Involvement in Churg-Strauss Syndrome
}

Yasmina Malky ${ }^{1 *}$, Khaoula Bourzeg ${ }^{1}$, Leila Chemaou El fihri ${ }^{1}$, Lamia Essaadouni ${ }^{2}$, Saloua El Karimi ${ }^{1}$, Mustapha El Hattaoui $^{1}$

${ }^{1}$ Cardiology Department, Mohammed VI University Hospital, Marrakesh, Morocco

${ }^{2}$ Department of internal medicine, Mohammed VI University Hospital, Marrakesh, Morocco

DOI: $10.36347 /$ sasjm.2022.v08i01.008

| Received: 09.12.2021 | Accepted: 14.01.2022 | Published: 24.01.2022

*Corresponding author: Yasmina Malky

Cardiology Department, Mohammed VI University Hospital, Marrakesh, Morocco

Abstract Original Research Article

Introduction: The Churg-Strauss syndrome is a very rare systemic disease affecting people between 40-60 years old. It is recognized by the combination of blood eosinophilia, eosinophilic tissue infiltration, extravascular fibrinoid necrotizing (allergic) epithelioid and eosinophilic granuloma formation, and disseminated necrotizing vasculitis occurring in asthmatic patients. The diagnosis is based on well-defined criteria. The relation between blood eosinophilia and heart disease is well recognized with very heterogeneous damage. Mortality is associated with the cardiac manifestation of CSS in $50 \%$ of case. We conducted the current study to determine the prevalence and clinical impact of cardiac involvement in CSS patients. Methods: This was a retrospective and descriptive study about 11 patients followed in the Department of Internal Medicine of CHU Mohamed VI for Churg Strauss syndrome between the year 2017 and 2021. Results: The average age of our patients was 50 years. The sex ratio was 0,57 . The main cardiovascular risk factors were age, male sex, diabetes and hypertension, with a history of rhinitis in two patients and thyroidectomy in one patient. The diagnosis of CHURG-STRAUSS was based on the diagnostic criteria established by the American College of Rheumatology. The echocardiography was the basic examination to objectify the cardiac involvement with the following results: detection of a pericardial effusion in 2 cases, dilated cardiomyopathy in one patient and severe alteration of the ventricular function in one patient. The extracardiac injuries were divided into renal and neurological injuries. Corticosteroid therapy with Endoxan was the cornerstone of the treatment, with non-specific treatment depending on the clinical presentation.

Keywords: Shurg-Strauss syndrome, cardiac involvement, perimyocarditis, heart failure, echocardiography, Cardiac magnetic resonance imaging.

Copyright $\left({ }^{2022}\right.$ The Author(s): This is an open-access article distributed under the terms of the Creative Commons Attribution 4.0 International License (CC BY-NC 4.0) which permits unrestricted use, distribution, and reproduction in any medium for non-commercial use provided the original author and source are credited.

\section{INTRODUCTION}

Churg-Strauss syndrome (CSS) is a necrotizing small-vessel vasculitis with extravascular granulomas and eosinophilic infiltrates [1]. The size of the targeted vessels and the occurrence of antineutrophil cytoplasmic antibodies (ANCA) led to its classification as an ANCA-associated systemic vasculitis. Involvement of the heart has been described in the third phase of CSS as vasculitic lesions in epicardial coronary vessels and in myocardium. It is a major cause of morbidity and mortality in these patients. Cardiomyopathy has long been recognized as the clinical correlate of endomyocardial fibrosis for most of the hypereosinophilic disorders. While Idiopathic hypereosinophilic syndrome, chronic eosinophilic leukemia, and tropical endomyocardial fibrosis all-share Loeffler endocarditis as the most severe cardiac manifestation causing high morbidity and mortality [2]. The treatment and prognosis of an individual CSS patient depends on the type and severity of organ manifestations.

\section{MATERIAL AND METHODS}

This is a retrospective and descreptive study, performed in the Department of Internal Medicine of the CHU Mohamed VI of Marrakech over a period of 04 years from January 2017 to December 2021. We retrieved all medical records, radiology and pathology reports of patients diagnosed with CSS from 2017 to 2021. Patients were entered in the study if data from initial presentation and at least 1 follow-up visit were available. Initially, 13 patients were screened. Two patients were excluded (missing data), resulting in 11 patients available for analysis. All patients fulfilled the 1990 American College of Rheumatology criteria [3]. 
Cardiac involvement of CSS was assumed in patients who had either clinical signs of acute cardiac failure, laboratory findings indicative for myocardial damage, or abnormalities in cardiac imaging such as echocardiography or cardiac MRI. Causes other than vasculitis had to be excluded. To investigate cardiac involvement, all patients had laboratory investigations of creatinine kinase, and a 12-lead electrocardiography (ECG) was recorded at diagnosis. Elevated creatinine kinase levels lead to analysis of troponin I. Echocardiography was performed in every patient with clinically suspected impaired cardiac function. Results were considered abnormal when regional or global systolic dysfunction, valvular abnormalities, pulmonary hypertension, or pericardial effusions were found.

\section{RESULTS}

We analyzed 11 patients with CSS. The mean age was 50 years (range, 32-67years).This series includes 4 men and 7 women with a sex ratio of 0.57 . In our study the diagnosis of Churg and Straus is retained in front of 4 criteria: Asthma (11 cases): $100 \%$, Blood eosinophilia $\geq 10 \%$ : (11 cases) $100 \%$, Mono- or polyneuropathy (7 cases): $63.63 \%$, Labile pulmonary infiltrates (5 cases): $45.45 \%$ (figure 1), Pain or sinus opacity (2 cases): $18.18 \%$, Presence of Extravascular eosinophil infiltrates were seen in $27.27 \%$ ( 3 cases) at biopsy. ANCA were found in 4 patients: 3 patients had p-ANCA with specificity for myeloperoxidase and 1 patient had c-ANCA specific for proteinase 3 .
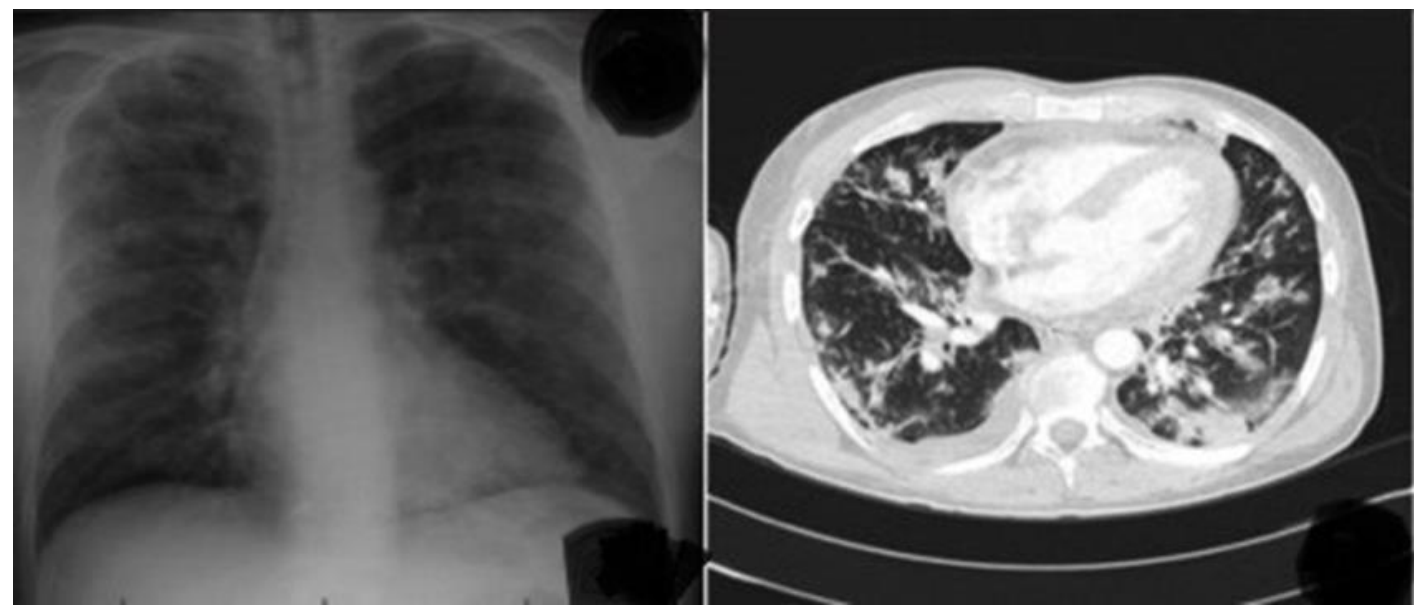

Fig-1: Chest X-ray and CT-scan showing alveolar condensation, pleural effusion, bronchial thickening and ground glass appearance

The profile of FRCVX in our patients was distributed as follows: age 5 cases: $45.45 \%$, male sex 4 cases: $36.36 \%$, menopause in 3 patients: $27.27 \%$, hypertension in only 3 cases: $27.27 \%$, diabetes 4 cases: $36.36 \%$ (graphic 1). For the ATCD 2 patients had rhinitis and one patient had undergone a thyroidectomy and was on Levothyrox.

In 3 patients, cardiac involvement was diagnosed by clinical signs of acute cardiac failure due to impaired left ventricular function. In two patients, the manifestation was dyspnea. All patients with cardiac involvement were ANCA-negative. ECG changes were found in 3 patients: right bundle branch block was identified in 1 patient, microvoltage with electrical alternation in 1 case and ventricular hypertrophy in 1 patient. Transthoracic echocardiography showed 4 cardiac lesions: a circumferential effusion with RV dysfunction in one patient, a posterior pericardial effusion without constriction image in one case, a dilated cardiomyopathy with severe global hypokinesia in one patients, severe impairment of LVEF in one patient.

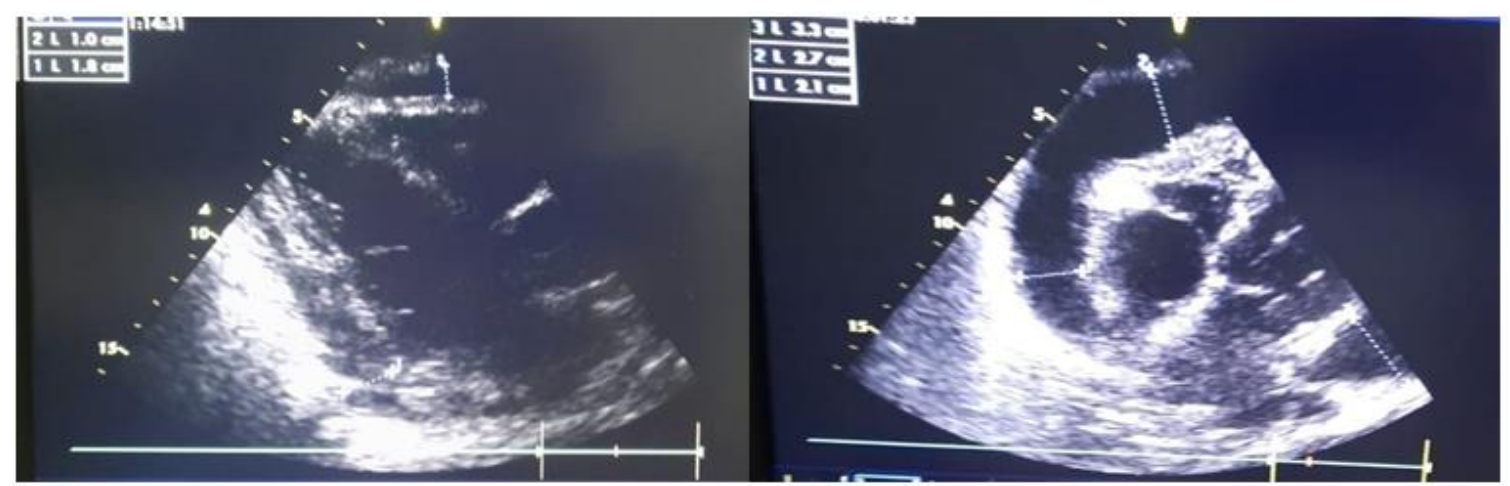

Fig-2: Circumferential effusion 
Renal involvement was noted in 4 patients. Neurological involvement in 6 patients was confirmed by electromyography (EMG). On therapeutic plant, all patients received 1 bolus of corticosteroid therapy followed by monthly oral boluses of endoxan-based immunosuppressant. With adjuvant therapy depending on the type of cardiac involvement. The overall mortality of our series is $0 \%$.

\section{DISCUSSION}

Churg-Strauss Syndrome (CSS) or "eosinophilic granulomatosis with polyangiitis" (EGPA) is a very rare systemic disease affecting people between 40-60 years old [4]. Typical lesions of CSS are histologically characterized by eosinophilia, necrotizing or granulomatous vascular lesions, and extravascular granulomas. The disease can be divided into 3 phases. The initial phase starts with allergic rhinitis, asthma, and nasal polyposis; the second is marked by peripheral and tissue eosinophilia with pulmonary infiltrates; the third is characterized by systemic vasculitis. Involvement of the heart has been described in the third phase of CSS as vasculitic lesions in epicardial coronary vessels and in myocardium [5]. CSS has an incidence of 0.11-2.66 cases per million per year and predominantly affects males between the third and sixth decade [5]. The most typical manifestations are allergic rhinitis, asthma, and peripheral blood eosinophilia. According to the American College of Rheumatology (ACR)[3], there are 6 criteria for the diagnosis of CSS: asthma, eosinophilia, pulmonary infiltrates, acute or chronic sinusitis, histological evidence of vasculitis with extravascular eosinophils, and mononeuropathy or polyneuropathy. The presence of 4 criteria allows the diagnosis to be made with a sensitivity of $\%$ and a specificity of 99.7 [3]. Serological markers of CSS are ANCA (essentially pANCA), which are prevalent in $40 \%$ of EGPA cases but less so in patients with cardiac involvement. About a quarter of cases have no peripheral eosinophilia [6]. Serum IgE levels are usually elevated, and IgG4 is found in $75 \%$ of all cases [7].

Cardiac manifestation of CSS has been reported with a prevalence of 66\% [8], however, postmortem studies suggest a prevalence of up to $92 \%$ [9]. In $50 \%$ of all CSS cases, mortality is associated with the cardiac manifestation of the disease presenting as progressive perimyocarditis, myocardial infarction or heart failure [6]. Studies suggested that heart involvement is more common in ANCA-negative patients [10]. The pathogenesis is eosinophilic infiltration, which rapidly progresses to fibrosis [11]. Acute left-ventricular dysfunction, signs of myocardial ischemia and arrhythmia are the main clinical features. Pericardial effusion secondary to pericarditis occurs in $20 \%$ of cases [11]. Compared to what has been referred to as idiopathic hypereosinophilic syndrome, endomyocarditis seems to be less common in CSS patients [11]. Apart from clinical assessment, screening cardiac involvement should include electrocardiography, echocardiography and plasma troponin measurement to detect myocardial ischemia. Echocardiography may show wall-motion abnormalities, signs of pericarditis or intraventricular thrombus. When coronary angiography is performed, stenoses, microaneurysms or thromboses are rarely observed because of the predominance of small vessel involvement [13]. Cardiac magnetic resonance imaging (MRI) provides a detailed anatomic description of the lesions; both first-pass deficits and late gadolinium enhancements are suitable to detect myocarditis and myocardial fibrosis. Therefore, active inflammation and fibrous changes are difficult to differentiate [12,9]. Dans le cadre d'une myocardiopathie, les biopsies endomyocardiques ne conduisent pas habituellement au diagnostic du CSS [13]. CSS patients with a pericardial effusion but preserved systolic left ventricular function had an excellent outcome and maintained cardiac function at follow-up. However, $27 \%$ of patients presented with severe cardiac manifestations, associated with reduced cardiac function in the majority of cases [11]. These patients had endomyocarditis as the underlying cause. Corticosteroids and long-term immunosuppression may improve event-free and disease-free survival rates in patients with cardiac manifestations of CSS [9].

\section{CONCLUSION}

Churg-Strauss syndrome is a condition whose spontaneous evolution was almost always fatal. Its prognosis has been transformed by the introduction of corticosteroids, then immunosuppressant.

\section{REFERENCES}

1. Churg, J., \& Strauss, L. (1951). Allergic granulomatosis, allergic angiitis, and periarteritis nodosa. The American journal of pathology, 27(2), 277.

2. Ogbogu, P. U., Rosing, D. R., \& Horne III, M. K. (2007). Cardiovascular manifestations of hypereosinophilic syndromes. Immunology and allergy clinics of North America, 27(3), 457-475.

3. Masi, A. T., Hunder, G. G., Lie, J. T., Michel, B. A., Bloch, D. A., Arend, W. P., ... \& Zvaifler, N. J. (1990). The American College of Rheumatology 1990 criteria for the classification of Churg-Strauss syndrome (allergic granulomatosis and angiitis). Arthritis \& Rheumatism, 33(8), 10941100 .

4. d'Ersu, E., Ribi, C., Monney, P., Vincenti, G., Schwitter, J., Rotman, S., ... \& Regamey, J. (2018). Churg-Strauss syndrome with cardiac involvement: case illustration and contribution of CMR in the diagnosis and clinical follow-up. International journal of cardiology, 258, 321-324.

5. Dendramis, G., Paleologo, C., Piraino, D., Arrotti, S., \& Assennato, P. (2015). Coronary involvement in Churg-Strauss syndrome. Indian heart journal, 67(6), 586. 
6. Brambatti, M., Matassini, M. V., Adler, E. D., Klingel, K., Camici, P. G., \& Ammirati, E. (2017). Eosinophilic myocarditis: characteristics, treatment, and outcomes. Journal of the American College of Cardiology, 70(19), 2363-2375.

7. Vaglio, A., Buzio, C., \& Zwerina, J. (2013). Eosinophilic granulomatosis with polyangiitis (C hurg-S trauss): state of the art. Allergy, 68(3), 261273.

8. Hazebroek, M. R., Kemna, M. J., Schalla, S., Sanders-van Wijk, S., Gerretsen, S. C., Dennert, R., ... \& Heymans, S. (2015). Prevalence and prognostic relevance of cardiac involvement in ANCA-associated vasculitis: eosinophilic granulomatosis with polyangiitis and granulomatosis with polyangiitis. International journal of cardiology, 199, 170-179.

9. Dennert, R. M., van Paassen, P., Schalla, S., Kuznetsova, T., Alzand, B. S., Staessen, J. A., ... \& Heymans, S. (2010). Cardiac involvement in Churg-Strauss syndrome. Arthritis \& Rheumatism: Official Journal of the American College of Rheumatology, 62(2), 627-634.
10. Gupta, S., Siddiqui, S., Haldar, P., Raj, J. V., Entwisle, J. J., Wardlaw, A. J., ... \& Brightling, C. E. (2009). Qualitative analysis of high-resolution CT scans in severe asthma. Chest, 136(6), 15211528.

11. Neumann, T., Manger, B., Schmid, M., Kroegel, C., Hansch, A., Kaiser, W. A., ... \& Zwerina, J. (2009). Cardiac involvement in Churg-Strauss syndrome: impact of endomyocarditis. Medicine, 88(4), 236-243.

12. Mahr, A., Moosig, F., Neumann, T., Szczeklik, W., Taillé, C., Vaglio, A., \& Zwerina, J. (2014). Eosinophilic granulomatosis with polyangiitis (Churg-Strauss): evolutions in classification, etiopathogenesis, assessment and management. Current opinion in rheumatology, 26(1), 16-23.

13. Isaka, N., Araki, S., Shibata, M., Takebayashi, S., Yada, T., Konishi, T., \& Nakano, T. (1994). Reversal of coronary artery occlusions in allergic granulomatosis and angiitis (Churg-Strauss syndrome). American heart journal, 128(3), 609613. 